\title{
Super-Resolution Microscopy: Spots, Speed, and Structure
}

\author{
* J.A. Galbraith and ** C.G. Galbraith \\ * NINDS, National Institutes of Health, Bethesda, MD 20892 \\ ** NICHD, National Institutes of Health, Bethesda, MD 20892
}

The dynamic three-dimensional scaffolds that organize both structure and function within every cell are made from ensembles of millions of molecules. Although live cell imaging of genetically expressed fluorescent probes has provided a glimpse into the workings of this subcellular realm, the resolution of light microscopy has restricted the level of our understanding to objects larger than the diffraction limit of $250 \mathrm{~nm}$. However, within a cell, thousands of molecules can be contained within a single diffraction-limited spot. Super-resolution microscopy [1-4] promises the opportunity to see the dynamic molecular inner workings of the cell.

The ultimate goal of super-resolution microscopy is to provide the speed and resolution to visualize and quantify the molecular mechanisms that control higher order cellular function. As we aim toward this goal, we establish new imaging milestones in molecular localization, threedimensional spatial resolution, and temporal acquisition. While each technological landmark offers the promise of allowing us to address biological questions that we never thought we would be able to ask much less answer; it also focuses our attention on the interrelationship the between the imaging parameters. Molecular specificity often costs localization precision. Spatial resolution demands high labeling density. Temporal resolution decreases as spatial resolution increases, and living cells have finite photon tolerances. Our goal is to continue to push these technologies toward high-speed, live-cell, multi-dimensional, molecular resolution microscopy.

Single molecule or localization super-resolution microscopy provides the resolution to localize individual molecules with great accuracy $(<20 \mathrm{~nm})$ in three-dimensional space. Individual molecular dynamics can be followed at high speed; however, the requirement of visualizing only a sub-set of non-overlapping molecules at any given time limits the temporal resolution for imaging molecular scaffolds. If cell can be imaged slowly, then three-dimensional superresolution of structures is possible if molecules can be labeled at a sufficiently high density. For $20 \mathrm{~nm}$ structural resolution an average molecular spacing of $10 \mathrm{~nm}$ is necessary [5]. This spacing corresponds to a density of 100 molecules / micron for a simple one-dimensional structure, but, since the number of molecules needed increases nonlinearly as the dimensionality increases, over $10^{\wedge} 6$ molecules / micron^ 3 are needed to accurately resolve a three-dimensional structure with $20 \mathrm{~nm}$ resolution. Therefore, the resolution of a super resolution image depends on the number of molecules localized not simply on the precision with which they are localized.

Applying single molecule superresolution techniques to study scaffolds and organelles in living cells is even more challenging. Sub-cellular scaffolds do not simply move; they also constantly change shape by gaining or losing molecules during image acquisition. Faster moving structures require more frequent sampling, which necessitates fewer localized molecules per image and potentially greater phototoxicity. Simply rendering single molecules with high precision does not guarantee a super resolution image of a dynamic structure. However, if we relax the 
requirements on spatial resolution, we can dramatically improve temporal resolution. Defined sheet illumination coupled with structured or two-photon illumination can be used to image cell volumes in $1 \mathrm{sec}$ with three-dimensional spatial resolution of less than the diffraction limit [6].

Each advance in spatial resolution or temporal resolution offers new opportunities. Each technological development has added to our understanding of molecular dynamics and how ensembles of molecules interact to form the structural and signaling scaffolds that direct cell function. The goal is to combine them to obtain high-speed, live-cell, multi-dimensional, molecular resolution microscopy to understand how molecules direct cell function.

\section{References}

[1] S.W. Hell, and J. Wichmann. Opt Lett. 19 (1994) 780.

[2] E. Betzig E., et al. Science. 313 (2006) 1642.

[3] M.J. Rust, M. Bates, and X. Zhuang. Nat. Meth. 3 (2006) 793.

[4] S.T. Hess, T.P. Girirajan, and M.D. Mason. Biophysical J. 91 (2006) 4258.

[5] Shannon, C. Proc IRE. 37 (1949) 10.

[6] Planchon, T., et al. Nat Meth. 2011

[7] This work was supported in part by the NINDS intramural research program 